\title{
Circulating leptin in non-alcoholic fatty liver disease: a systematic review and meta-analysis
}

\author{
Stergios A. Polyzos ${ }^{1}$ (D) - Konstantinos N. Aronis ${ }^{2,3}$ • Jannis Kountouras ${ }^{1} \cdot$ \\ Dimitrios D. Raptis ${ }^{1} \cdot$ Maria F. Vasiloglou ${ }^{1}$ - Christos S. Mantzoros ${ }^{2,3,4}$
}

Received: 17 May 2015 / Accepted: 7 September 2015 / Published online: 26 September 2015

(C) Springer-Verlag Berlin Heidelberg 2015

\begin{abstract}
Aims/hypothesis Clinical data regarding circulating leptin levels in patients with non-alcoholic fatty liver disease (NAFLD) are conflicting. The purpose of this meta-analysis was to compare leptin levels between the following groups: patients with biopsy-proven NAFLD vs controls; simple steatosis (SS) patients vs controls; non-alcoholic steatohepatitis (NASH) patients vs controls and NASH patients vs SS patients.

Methods We performed a systematic search in PubMed, Scopus and the Cochrane Library. We analysed 33 studies, published between 1999 and 2014, including 2,612 individuals (775 controls and 1,837 NAFLD patients).

Results Higher circulating leptin levels were observed in NAFLD patients vs controls (standardised mean difference [SMD] 0.640; 95\% CI 0.422, 0.858), SS patients vs controls (SMD $0.358 ; 95 \%$ CI $0.043,0.673$ ), NASH patients vs controls (SMD 0.617; 95\% CI 0.403, 0.832) and NASH patients
\end{abstract}

Electronic supplementary material The online version of this article (doi:10.1007/s00125-015-3769-3) contains peer-reviewed but unedited supplementary material, which is available to authorised users.

Stergios A. Polyzos

stergios@endo.gr

Second Medical Clinic, Department of Medicine, Aristotle

University of Thessaloniki, Ippokration Hospital, 49

Konstantinoupoleos, 54642 Thessaloniki, Greece

2 Division of Endocrinology, Diabetes and Metabolism, Department of Internal Medicine, Beth Israel Deaconess Medical Center, Harvard Medical School, Boston, MA, USA

3 Department of Medicine, Boston Medical Center, Boston University School of Medicine, Boston, MA, USA

4 Section of Endocrinology, Boston VA Healthcare System, Harvard Medical School, Boston, MA, USA vs SS patients (SMD 0.209; 95\% CI 0.023, 0.395). These results remained essentially unchanged after excluding studies involving paediatric or adolescent populations and/or individuals undergoing bariatric surgery. There was moderate-tosevere heterogeneity among studies in all comparisons, but no significant publication bias was detected. Meta-regression analysis demonstrated that BMI was inversely associated with leptin SMD and accounted for $26.5 \%(p=0.014)$ and $32.7 \%$ $(p=0.021)$ of the between-study variance in the comparison between NASH patients and controls and NAFLD patients and controls, respectively. However, when bariatric studies were excluded, BMI did not significantly explain the between-study variance.

Conclusions/interpretation Circulating leptin levels were higher in patients with NAFLD than in controls. Higher levels of circulating leptin were associated with increased severity of NAFLD, and the association remained significant after the exclusion of studies involving paediatric or adolescent populations and morbidly obese individuals subjected to bariatric surgery.

Keywords Adipokine - Adipose tissue $\cdot$ Insulin resistance Leptin · Meta-analysis $\cdot$ Metabolic syndrome $\cdot$ Non-alcoholic fatty liver disease $\cdot$ Non-alcoholic steatohepatitis $\cdot$ Steatosis . Systematic review

$\begin{array}{ll}\text { Abbreviations } \\ \text { ALT } & \text { Alanine aminotransferase } \\ \text { AST } & \text { Aspartate aminotransferase } \\ \text { GGT } & \gamma \text {-Glutamyltranspeptidase } \\ \text { IR } & \text { Insulin resistance } \\ \text { IRMA } & \text { Immunoradiometric assay } \\ \text { MD } & \text { Mean difference } \\ \text { NAFLD } & \text { Non-alcoholic fatty liver disease }\end{array}$




$\begin{array}{ll}\text { NAS } & \text { NAFLD activity score } \\ \text { NASH } & \text { Non-alcoholic steatohepatitis } \\ \text { NOS } & \text { Newcastle-Ottawa scale } \\ \text { PRISMA } & \begin{array}{l}\text { Preferred Reporting Items for Systematic reviews } \\ \text { and Meta-Analyses }\end{array} \\ \text { SMD } & \begin{array}{l}\text { Standardised MD } \\ \text { SS }\end{array} \\ \text { Simple steatosis }\end{array}$

\section{Introduction}

Non-alcoholic fatty liver disease (NAFLD) is thought to be the hepatic component of the metabolic or insulin resistance (IR) syndrome; its prevalence is increasing worldwide in parallel with the obesity epidemic and increasing prevalence of type 2 diabetes mellitus [1]. NAFLD ranges from nonalcoholic simple steatosis (SS) to non-alcoholic steatohepatitis (NASH) characterised by steatosis, inflammation and fibrosis. NAFLD is a global public health problem that, apart from its hepatic consequences (liver cirrhosis and/or hepatocellular carcinoma), has systemic complications related to IR syndrome and cardiovascular diseases [2].

Leptin, a $16 \mathrm{kDa}$ protein, is the first adipose-tissue-secreted hormone (adipokine) to be described. Leptin is secreted proportionally to the amount of white adipose mass and is present in the circulation either as the free form or bound to proteins. Its levels in the circulation and adipose tissue depend on the amount of adipose tissue and the status of energy balance; circulating leptin levels reflect primarily body energy stores and secondarily acute changes in energy intake [3]. Leptin plays a crucial role in regulation of energy homeostasis, glucose and lipid metabolism, reproduction and neuroendocrine function $[4,5]$. There is also emerging evidence that leptin is involved in cognition, immune function and bone metabolism [6].

IR and adipokines contribute to the pathogenesis of SS and the progression from SS to NASH and NASH-related cirrhosis [1]. However, the specific mechanisms involved remain to be elucidated and the role of leptin in the pathogenesis and progression of NAFLD remains largely unknown. We had previously hypothesised that leptin may have an antisteatotic effect but that excessive levels may result in hepatic inflammation and fibrosis [7]. Experimental data have recently shown that whereas leptin deficiency may lead to hepatic steatosis (e.g. in $o b / o b$ mice), leptin excess contributes to hepatic inflammation and fibrosis [8]. In this regard, its potential application in patients with NAFLD and NASH will require additional understanding of leptin's role in lipid handling, hepatic inflammation and fibrosis, along with identification of the potential subsets of patients who might benefit.

Clinical data regarding circulating leptin levels in NAFLD patients are conflicting, with some authors reporting higher levels in NAFLD patients than in controls and others reporting similar levels among groups [9]. Elucidating the role of leptin in NAFLD might have clinical implications, because of the need for non-invasive biomarkers for NASH and NASHtargeting treatment $[10,11]$.

The aim of this systematic review and meta-analysis was to summarise and compare existing data on circulating leptin levels in biopsy-proven patients with NAFLD (SS and/or NASH) and controls. A deeper insight into the interplay between leptin and NAFLD may lead to the design of new clinical trials aimed at elucidating the potential prognostic and/or therapeutic roles of leptin in NAFLD.

\section{Methods}

Literature search This systematic review was conducted following an a priori established protocol and is reported according to the Meta-analysis of Observational Studies in Epidemiology (MOOSE) statement [12]. We performed a computerised literature search using the PubMed, Scopus and Cochrane Library electronic databases. The search was not limited by language or publication time. The Medical Subject Heading (MeSH) database was used as a terminological search filter. From the combination of terminological (MeSH terms) and methodological search filters, as proposed elsewhere in detail [13], the following query was formatted: 'leptin AND (NAFLD OR NASH OR [nonalcoholic fatty liver disease] OR [nonalcoholic steatohepatitis] OR [non-alcoholic fatty liver disease] OR [non-alcoholic steatohepatitis])' This query was used with minimal differences, according to the requirements of different databases. The full query used in PubMed can be found in electronic supplementary material (ESM) Methods. The literature search was extended by manual search in the 'related citations' links of all included articles in PubMed, and the references of all included articles [13].

Inclusion and exclusion criteria Clinical studies of any design providing comparative circulating leptin levels for NAFLD (or SS or NASH) patients and/or controls were eligible for this systematic review and meta-analysis. Studies were included in the systematic review and meta-analysis, if they met the following criteria: (1) they were original full-text publications; (2) they included biopsy-proven NAFLD patients and (3) they included comparison of circulating leptin levels between NAFLD patients and NAFLD-free controls.

Studies were excluded from the systematic review and meta-analysis on the following grounds: (1) inclusion of patients with other causes of liver disease (e.g. alcoholic fatty liver disease, viral or autoimmune hepatitis) or in whom NAFLD co-existed with other liver disease(s); (2) there was overlap of patients (i.e. the same participants were included in more than one study); (3) additional data were needed, but 
corresponding authors did not respond; (4) studies were of low methodological quality as defined by a Newcastle-Ottawa scale (NOS) score $\leq 2 ;$ (5) studies were interventional with similar comparative groups at baseline (e.g. all groups had SS or NASH at baseline); (6) retrieved articles were reviews, editorials, case reports, letters to the editor, hypotheses, book chapters, studies on animals or cell lines, although letters to the editor providing original data were considered; and (7) unpublished data or abstracts from conferences. Only fulltext articles were included, the quality of which is more easily assessed.

In studies that had more than one control group, we included the control group whose characteristics were more similar to the patient group(s), giving priority to BMI and HOMA-IR. For example, if there was one obese and one lean control group and NAFLD patients were obese in a specific study, we included the obese control group in the meta-analysis.

Data extraction All retrieved articles were saved in an EndNote file (Thompson Reuters, Philadelphia, PA, USA) to facilitate their handling. Two experienced reviewers (SAP and $\mathrm{JK})$, qualified in systematic review and meta-analysis, reviewed independently the title and abstract of all initial search results and excluded studies that did not address the research question, based on our pre-specified inclusion and exclusion criteria. If multiple publications from the same cohort were found, only data from the largest comprehensive study were included. For articles published in a language other than English, a list of required items was requested from the corresponding authors. Subsequently, review of full-text articles and quality assessment was independently carried out by the aforementioned reviewers. A third reviewer (CSM), who was not involved in the previous literature screening and search, was available to adjudicate on any disagreement between the two reviewers.

We extracted the following variables from each study: (1) the study's general characteristics (reference, name of the first author; year of publication; country where the study was carried out; design); (2) the study's special characteristics (paediatric/adolescent population; morbidly obese population subjected to bariatric surgery; liver biopsy on the controls; inclusion of patients with NASH-related cirrhosis); (3) patient and/ or control characteristics (number, sex, age, BMI, frequency of type 2 diabetes); (4) methods used to evaluate leptin levels and classify NAFLD patients; (5) biochemical measurements (leptin, aspartate aminotransferase [AST], alanine aminotransferase $[\mathrm{ALT}]$ and $\gamma$-glutamyltranspeptidase [GGT] levels) and (6) evaluation of IR (HOMA-IR).

All corresponding (and/or first authors) of the included studies were contacted by e-mail to request additional data or to request data transformations. Conflicts of data extraction were resolved by consensus after discussion and/or referral back to the corresponding (or first) authors. When corresponding (or first) authors did not respond, transformations were made by standard formulas. If the additional data were considered to be of importance (e.g. many missing values, transformations by formulas not feasible, the performance of liver biopsy, unknown, etc.), and the corresponding author did not respond or was unwilling to provide additional data, the study was excluded from the meta-analysis.

Quality assessment The quality assessment was performed independently by two reviewers (SAP and JK) using the NOS (Ottawa Hospital Research Institute, Ottawa, ON, Canada). The chance-adjusted inter-rater agreement between the two reviewers was evaluated by the Cohen's $k$ test. The mentioned third reviewer (CSM) was not involved in the initial quality assessment, but was again available to adjudicate on any disagreement between the two reviewers. The NOS is an instrument that has been developed to assess the quality of observational studies and is frequently used in meta-analyses. It evaluates study validity across three domains: the selection of the study groups; the comparability of the groups and the assessment of the outcome. The quality of the studies is rated on a scale from 0 (very poor) to 9 (high).

Outcomes The main outcome of the meta-analysis was the standardised mean difference (SMD) of circulating leptin levels between NAFLD patients and NAFLD-free controls. NAFLD patients were further classified as SS or NASH based on Brunt [14] or NAFLD activity score (NAS) [15] histological systems in most studies. Subsequently, we compared leptin levels between the following groups: (1) NAFLD patients vs controls; (2) SS patients vs controls; (3) NASH patients vs controls and (4) NASH patients vs SS patients.

Statistical analysis The statistical analysis was performed with R3.03 software (R Development Core Team; https:// www.r-project.org) and the 'meta' package. Given the expected heterogeneity of the outcome, we primarily analysed our data with a random-effects inverse-variance meta-analysis, through the 'metacont' routine. We also reported the results from fixed-effects inverse-variance models. We expressed the effect size of the meta-analysis as SMD of leptin levels, and Hedges' $g$ was used to pool variances for the standardisation. The heterogeneity of the outcome was evaluated using the $I^{2}$ statistic. We assessed the existence of any influential study that could be driving the result of the metaanalysis with the 'metainf' routine.

We also examined potential publication bias. Publication bias was defined as the tendency of authors and editors to handle studies in which the experimental results achieved statistical significance more favourably when compared with studies in which the results fail to reach significance, which ultimately introduces bias in the literature. We assessed the existence of publication bias with funnel plots and quantified 
funnel plot asymmetry with Egger's test for small-study effects, through the 'metabias' routine. The Egger's test performs a linear regression of the SMDs on their standard errors, weighting by the inverse variance of the SMD and testing the null hypothesis of no small-study effect (i.e. no funnel plot asymmetry).

Moreover, we performed univariate, random-effects metaregression analysis on aggregate-level data in which the SMD was regressed on the following factors: (1) whether or not the diagnosis of controls was biopsy-proven; (2) whether or not patients with NASH-related cirrhosis were included; (3) percentage of male sex; (4) percentage of type 2 diabetes; (5) mean age; (6) mean BMI and (7) mean HOMA-IR of each study's cohort. Regression coefficients ( $\beta$ ) with their $p$ values, and coefficients of determination $\left(R^{2}\right.$, as an index of the heterogeneity accounted for in the model) are reported. All $p$ values were two sided. The $\alpha$ criterion was set at 0.05 .
We performed the above-mentioned analysis in the sum of included studies, and then we performed a sensitivity analysis by excluding studies reporting on paediatric/adolescent populations or morbidly obese adults subjected to bariatric surgery, or both. As another sensitivity analysis, we repeated the analyses by using leptin mean difference (MD) instead of SMD.

\section{Results}

Literature search We initially retrieved 435 articles from PubMed, 841 from Scopus and 11 from Cochrane Library (last update 1 January 2015). Sixteen additional articles were added from the 'related citations' links and the references of the selected articles. A flowchart, which was prepared according to the Preferred Reporting Items for Systematic reviews
Fig. 1 Flowchart presenting the literature search process, according to the PRISMA statement
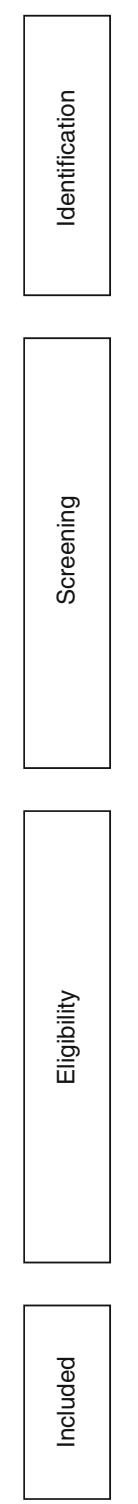

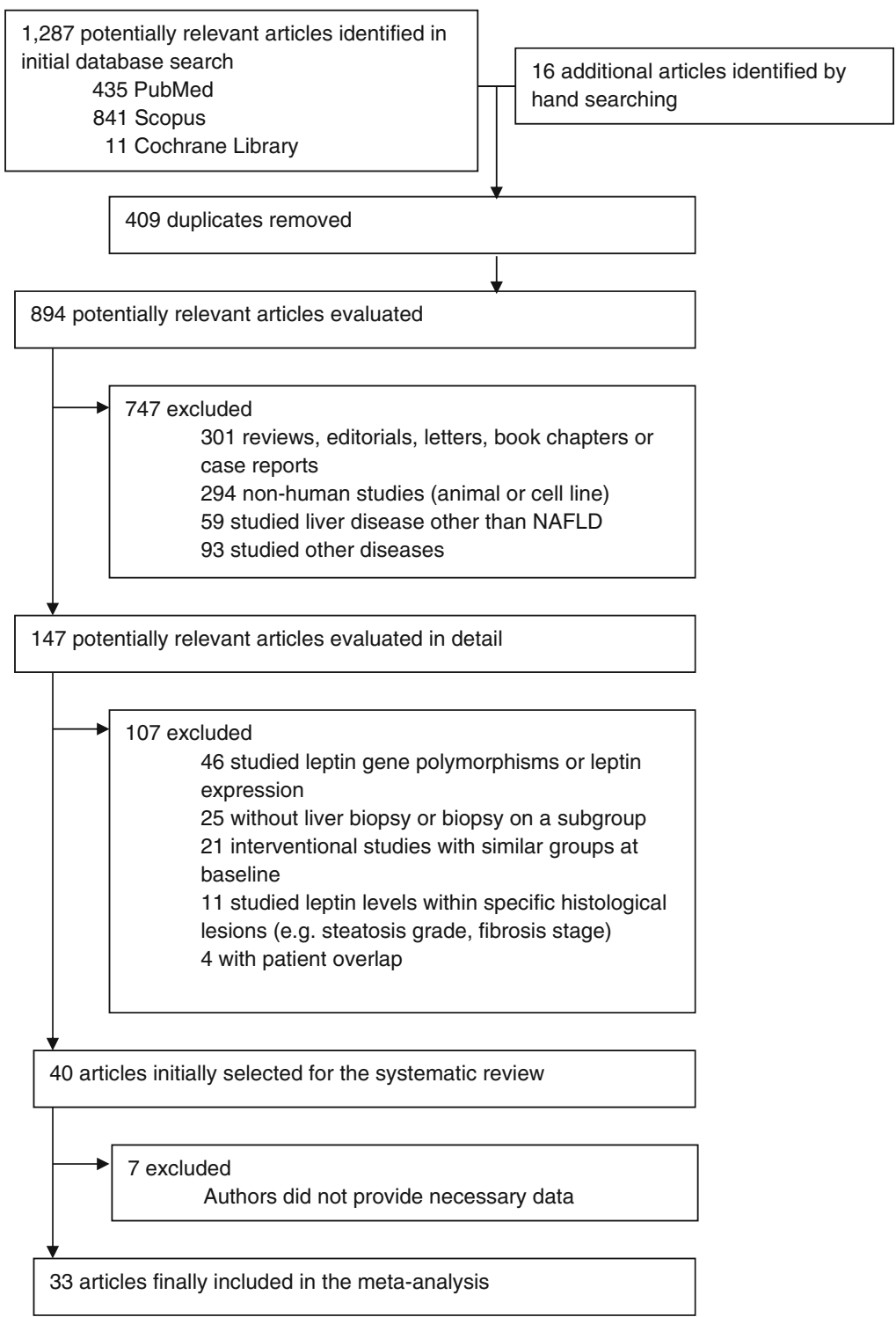




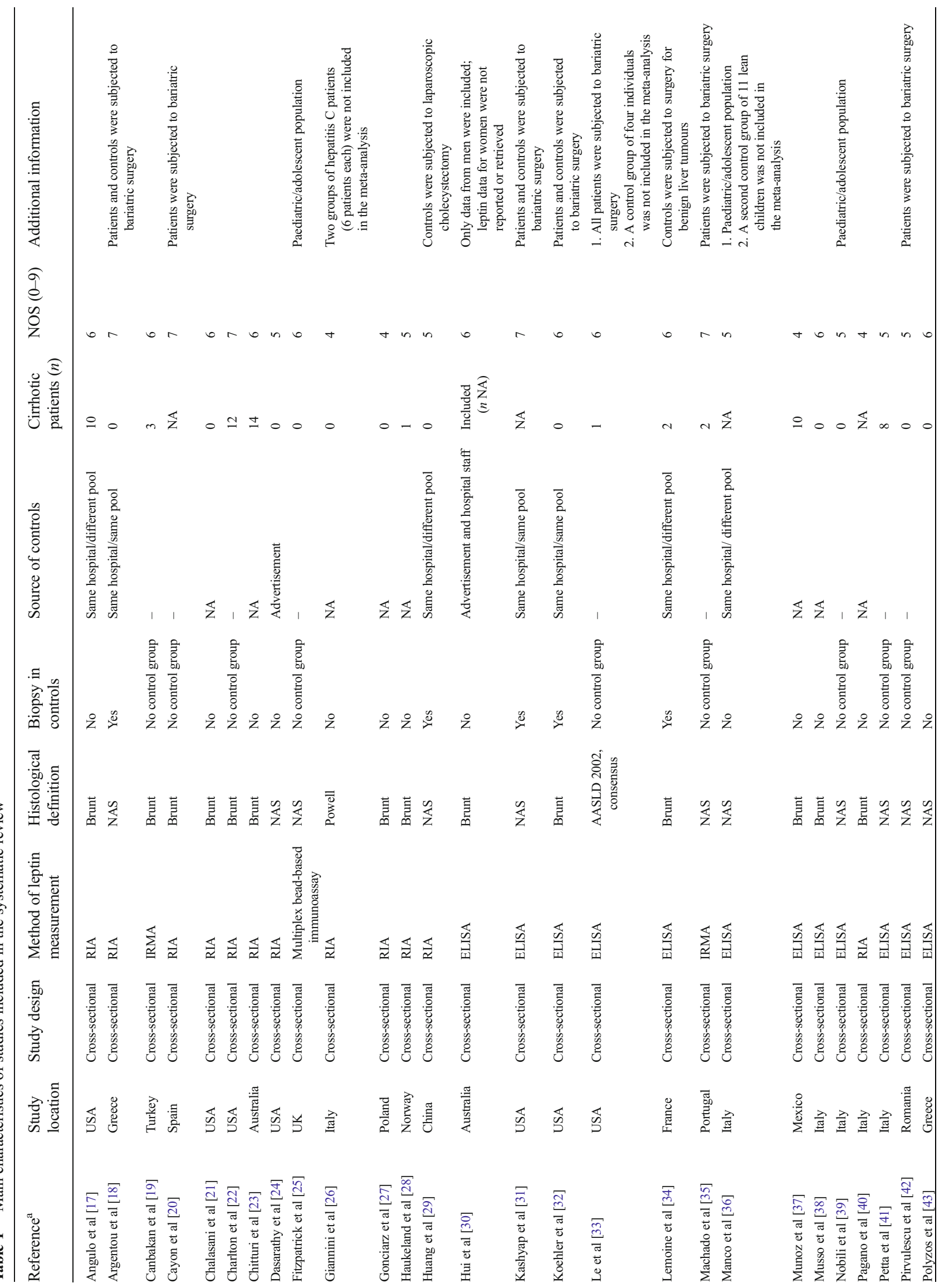




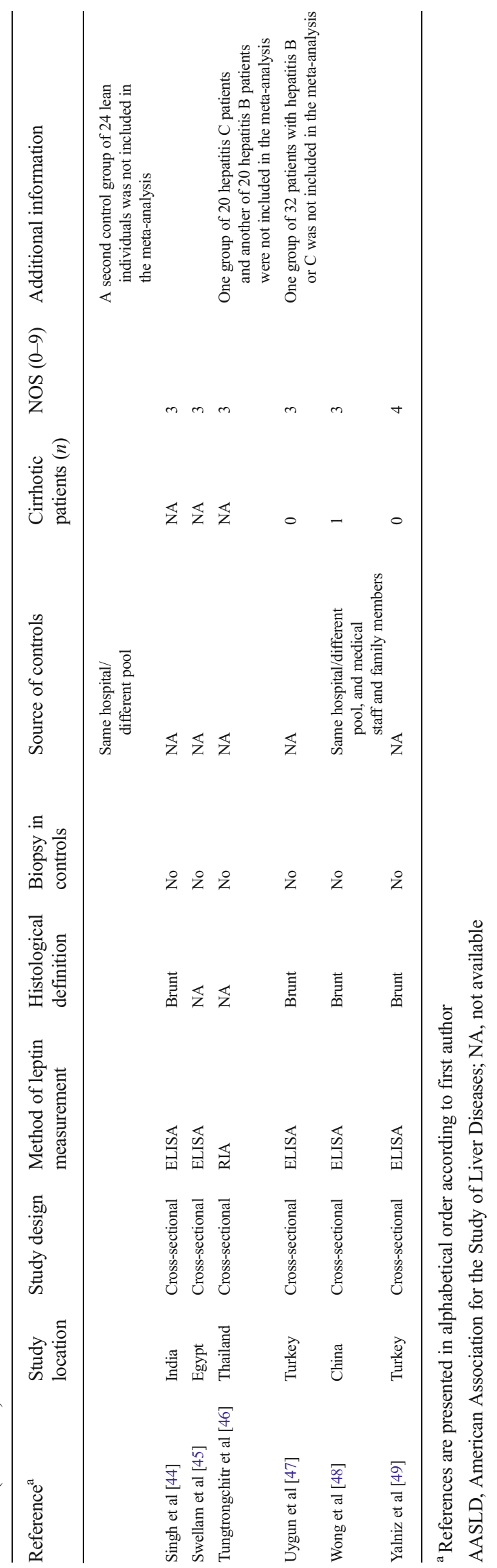


and Meta-Analyses (PRISMA) guidelines [16], summarises the identification, screening, eligibility and final selection of the studies included in the meta-analysis (Fig. 1). Forty studies were initially considered eligible for this meta-analysis. We tried to communicate with the corresponding (and/or first) authors of all but one of them (our study), to request additional data. Sixteen of the corresponding (or first) authors provided us with the requested additional/transformed data, whereas 23 authors did not respond $(n=22)$ or were unwilling to provide additional data $(n=1)$; seven studies were excluded at the final step because the requested data were considered to be absolutely necessary (ESM Table 1). At the end of the process, 33 studies were included in the meta-analysis.

Characteristics of the included studies The 33 included studies were published between 1999 and 2014, and reported data from 2,612 individuals (775 controls and 1,837 NAFLD patients) [17-49]. The main characteristics of these studies are presented in Table 1. In 1999, Giannini et al published the first study comparing leptin levels in individuals with biopsyproven NASH (six men) vs controls (six) [26]. Fifteen studies were carried out in Europe, seven in North America, seven in Asia, two in Australia, one in South America and one in Africa. All studies included in the meta-analysis were crosssectional. All identified interventional studies had similar comparative groups at baseline (usually NASH) and were excluded. Liver biopsies on controls were performed in five studies. Circulating leptin levels were measured by ELISA in 17 studies, RIA in 13 studies, immunoradiometric assay (IRMA) in two studies and a bead-based immunoassay in one study.

The main demographic and biochemical characteristics per group of each study included in the meta-analysis are presented in ESM Table 2. We reported circulating leptin levels, sex, age, BMI, number of type 2 diabetes patients, liver function tests (AST, ALT and GGT levels) and the HOMA-IR. Data for NAFLD patients were not provided if there was not a control group in the study (i.e. when the study compared leptin levels between SS and NASH patients), since a comparison between NAFLD patients and controls could not be performed. Nine studies included all groups (controls, SS, NASH patients) [18, 28, 30-32, 34, 40, 43, 48]; 11 studies compared leptin levels between NASH patients and controls, without recruiting an SS group [21, 23, 24, 26, 27, 37, 38, 44, 46, 47, 49]; four studies compared leptin levels between NAFLD patients and controls, without providing separate data for SS and NASH $[17,29,36,45]$ and nine studies compared leptin levels between SS and NASH patients, without recruiting a control group [19, 20, 22, 25, 33, 35, 39, 41, 42] (ESM Table 2). Subsequently, comparative data was provided as follows: 24 studies, NAFLD patients $(n=1,231)$ vs controls $(n=775)$; nine studies, SS patients $(n=283)$ vs controls $(n=313) ; 20$ studies, NASH patients $(n=699)$ vs controls $(n=627)$ and 18 studies, SS $(n=618)$ vs NASH patients $(n=702)$.

Quality of included studies The quality of included studies according to NOS is presented in Table 1 . The chanceadjusted inter-rater agreement between the two reviewers was satisfactory (Cohen's $k=0.89 ; p<0.001$ ). According to NOS results, five studies were scored 3 , five studies 4 , seven studies 5, 11 studies 6 and five studies 7 (mean \pm SD 5.2 \pm 1.3 ). No study was scored $\leq 2$, so no study was excluded on the basis of poor NOS score.

Outcomes Higher circulating leptin levels were observed in the following groups: (1) NAFLD patients vs controls; (2) SS patients vs controls; (3) NASH patients vs controls and (4) NASH vs SS patients (Table 2; Fig. 2). There was moderateto-severe heterogeneity among studies in all comparisons ( $I^{2}$ ranged from $59.4 \%$ to $77.6 \%$; Fig. 2). No sign of publication bias was detected in any comparison ( $p>0.05$ for all comparisons; Table 3 and ESM Fig. 1).

In the sensitivity analysis, after excluding paediatric/ adolescent studies ( $n=3$; ESM Fig. 2 ) or bariatric studies

Table 2 Comparison between groups in the sum of included studies, and after exclusion of paediatric/adolescent studies or studies of morbidly obese adults subjected to bariatric surgery or both

\begin{tabular}{cllll}
\hline Comparison & All studies & Excluding paediatric/adolescent studies & Excluding bariatric studies & $\begin{array}{l}\text { Excluding both paediatric/adolescent } \\
\text { and bariatric studies }\end{array}$ \\
\hline NAFLD vs control & $0.64(0.42,0.86)$ & $0.64(0.41,0.87)$ & $0.72(0.50,0.95)$ & $0.73(0.49,0.96)$ \\
$\quad p$ value & $<0.0001$ & $<0.0001$ & $<0.0001$ & $<0.0001$ \\
SS vs control & $0.36(0.04,0.67)$ & $0.36(0.04,0.67)$ & $0.50(0.08,0.91)$ & $0.50(0.08,0.91)$ \\
$\quad p$ value & 0.026 & 0.026 & 0.019 & 0.019 \\
NASH vs control & $0.62(0.40,0.83)$ & $0.62(0.40,0.83)$ & $0.72(0.51,0.94)$ & $0.72(0.51,0.94)$ \\
$p$ value & $<0.0001$ & $<0.0001$ & $<0.0001$ & $<0.0001$ \\
NASH vs SS & $0.21(0.02,0.40)$ & $0.21(0.07,0.36)$ & $0.15(-0.17,0.46)$ & $0.21(0.03,0.39)$ \\
$\quad p$ value & 0.028 & 0.004 & 0.367 & 0.019 \\
\hline
\end{tabular}

Data are presented as SMD $(95 \% \mathrm{CI})$ 
Fig. 2 Forest plots presenting the quantitative synthesis of circulating leptin levels, comparing the following groups in the sum of the included studies: NAFLD patients vs controls (a); SS patients vs controls (b);

NASH patients vs controls (c) and NASH patients vs SS patients (d). $\mathrm{W}$, weight

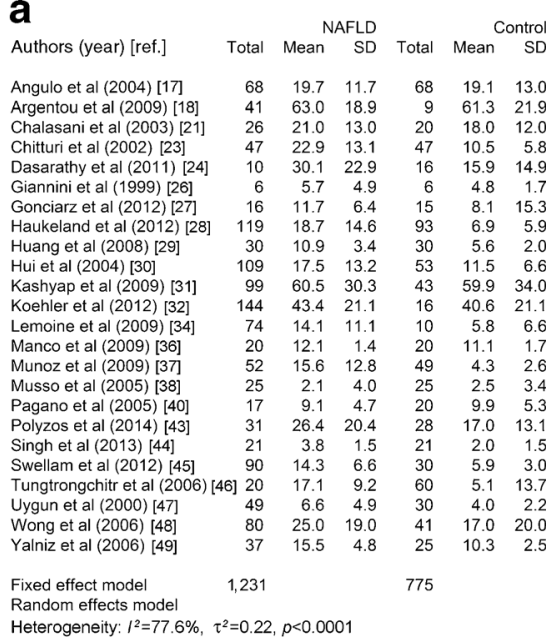

b
SD Total Mean ${ }^{\text {Control }}$ SD

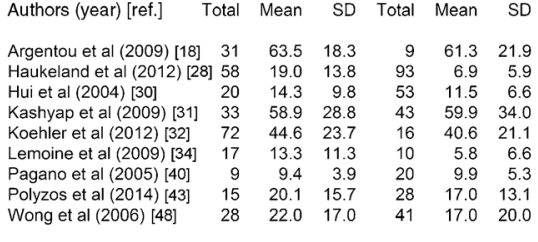

Random effects model
Heterogeneity: $I^{2}=71.9 \%, \tau^{2}=0.14, p=0.0004$

C

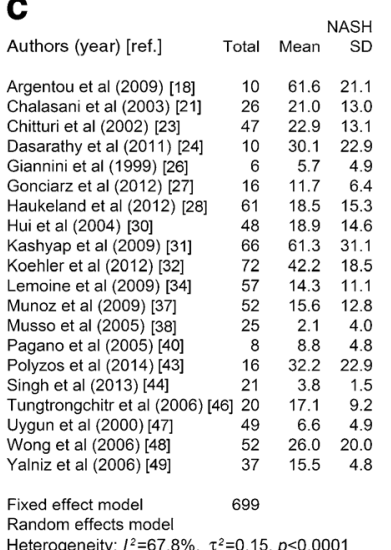

Heterogeneity: $I^{2}=67.8 \%, \tau^{2}=0.15, p<0.0001$

d Authors (year) [ref.] Total Mean SASH Total Mean SS Argentou et al (2009) [18] $\quad 10 \quad 61.6 \quad 21.1$ Canbakan et al (2008) [19] $35 \quad 52.6 \quad 36.8$ $\begin{array}{llll}\text { Canbakan et al (2008) [19] } & 35 & 52.6 & 36.8 \\ \text { Cayon et al (2006) [20] } & 55 & 50.1 & 21.2\end{array}$ Chariton et al (2008) [22] $\quad 25 \quad 33.920 .6$ Fitzpatrick et al (2012) [25] $35 \quad 41.4 \quad 20.4$ Haukeland et al (2012) [28] $61 \quad 18.5 \quad 15.3$ $\begin{array}{llll}\text { Hui et al (2004) [30] } & 48 & 18.9 & 14.6\end{array}$ Kashyap et al (2009) [31] $\quad \begin{array}{llll}66 & 61.3 & 31.1\end{array}$ Koehler et al (2012) [32] $\quad \begin{array}{llll}72 & 42.2 & 18.5\end{array}$ $\begin{array}{lllr}\text { Le et al (2007) [33] } & 21 & 11.5 & 3.4\end{array}$ Lemoine et al (2009) [34] $\quad 57 \quad 14.3 \quad 11.1$ \begin{tabular}{llll} 
Machado et al (2012) [35] & 11 & 22.2 & 6.8 \\
\hline
\end{tabular} \begin{tabular}{lrrr} 
Nobili et al (2006) [39] & 46 & 18.7 & 7.1 \\
\hline
\end{tabular} $\begin{array}{lrrr}\text { Pagano et al (2005) [40] } & 8 & 8.8 & 4.8 \\ \text { Petta et al (2012) [41] } & 71 & 18.2 & 13.8\end{array}$ $\begin{array}{llll}\text { Petta et al (2012) [41] } & 71 & 18.2 & 13.8 \\ \text { Pirvulescu et al (2012) [42] } & 13 & 59.8 & 32 .\end{array}$ Pirvulescu et al (2012) [42] $13 \quad 59.8 \quad 32.9$ $\begin{array}{llll}\text { Polyzos et al (2014) [43] } & 16 & 32.2 & 22.9 \\ \text { Wong et al (2006) [48] } & 52 & 26.0 & 20.0\end{array}$$$
\text { Fixed effect model } \quad 702
$$

Random effects model
Heterogeneity: $l^{2}=59.4 \%, \tau^{2}=0.08, p=0.0007$

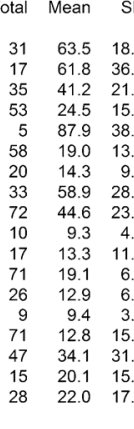

SD

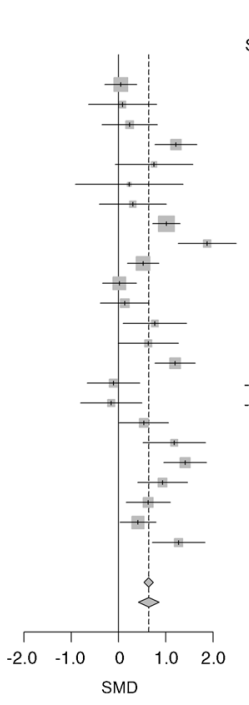

SMD $\quad 95 \% \mathrm{Cl} \quad \mathrm{W}$, fixed $\mathrm{W}$, random

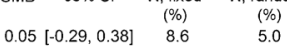
$0.09[-0.64,0.81]$ $0.23[-0.35,0.82]$ $0.75[-0.07,1.57$ $0.23[-0.91,1.36$ $0.30[-0.41,1.01]$ $1.01[0.72,1.30]$

$1.88[1.26,2.49]$ $0.52[0.19,0.85]$ $0.02[-0.34,0.38]$ $0.13[-0.38,0.65]$ $0.77[0.10,1.44]$ $1.20[0.77,1.62]$ $-0.11[-0.66,0.45]$ $-0.16[-0.80,0.49]$ $0.54[0.01,1.06]$ $1.18[0.52,1.84]$ $1.41[0.96,1.86]$ $0.93[0.40,1.46]$ $0.63[0.16,1.09]$ $0.41[0.03,0.79]$ $0.64[0.54,0.74] \quad 100$ $0.64[0.42,0.86]$

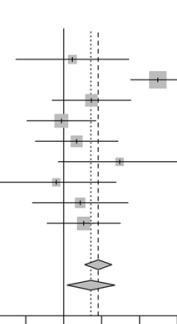

SMD $\quad 95 \% \mathrm{Cl}$ W fixed $W$ random (\%) $1.24[0.88,1.60] 24$ $0.37[-0.15,0.88] \quad 11.8$ $-0.03[-0.48,0.42] \quad 15.4$ $\begin{array}{lll}0.17[-0.37,0.71] & 10.8 & 11.7\end{array}$ $\begin{array}{rrr}0.74[-0.07,1.55] & 4.8 & 8.2 \\ -0.10[-0.89,0.69] & 5.1 & 8.4\end{array}$ $\begin{array}{rrr}-0.10[-0.89,0.69] & 5.1 & 8.4 \\ 0.22[-0.41,0.85] & 8.0 & 10.4\end{array}$ $0.26[-0.22,0.74] \quad 13.6$

$0.45[0.28,0.63] \quad 100$ $0.36[0.04,0.67]$

100

$\begin{array}{lllllll}-1.5 & -1.0 & -0.5 & 0 & 0.5 & 1.0 & 1.5\end{array}$

SMD
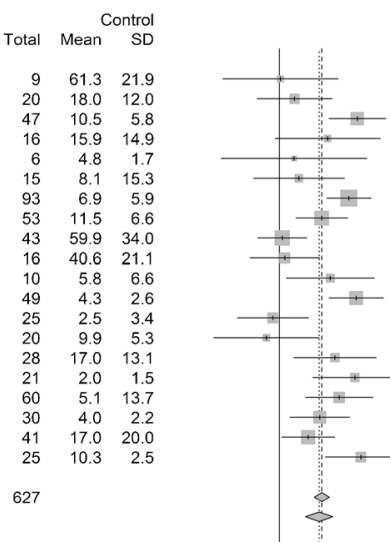
SMD $\quad 95 \% \mathrm{Cl}$ $0.01[-0.89,0.91]$ $0.23[-0.35,0.82]$ $0.75[-0.07,1.57]$ $0.23[-0.91,1.36]$ $0.30[-0.41,1.01]$ $1.08[0.74,1.43]$ $0.66[0.26,1.06]$ $0.04[-0.34,0.43]$ $0.08[-0.46,0.63]$ $0.79[0.11,1.48]$ $1.20[0.77,1.62]$ $-0.11[-0.66,0.45]$ $-0.21[-1.03,0.62]$
$0.87[0.22,1.51]$ $0.87[0.22,1.51]$ $0.93[0.40,1.46]$ $0.63[0.16,1.09]$ $1.27[0.71,1.83]$

$0.66[0.54,0.78]$

\begin{tabular}{|c|c|}
\hline $\begin{array}{l}\text { W, fixed } \\
(\%)\end{array}$ & $\begin{array}{l}W, \text { random } \\
(\%)\end{array}$ \\
\hline 1.7 & 3.3 \\
\hline 4.0 & 5.0 \\
\hline 7.1 & 5.9 \\
\hline 2.0 & 3.7 \\
\hline $\begin{array}{l}1.1 \\
\text { S }\end{array}$ & 2.5 \\
\hline 2.7 & 4.3 \\
\hline 11.5 & $\begin{array}{l}6.06 \\
6.6\end{array}$ \\
\hline 8.5 & 6.2 \\
\hline 9.3 & 6.3 \\
\hline 4.7 & 5.3 \\
\hline 2.9 & 4.4 \\
\hline 7.6 & 6.1 \\
\hline 4.5 & 5.2 \\
\hline 2.0 & 3.7 \\
\hline 3.3 & 4.6 \\
\hline 3.2 & 4.5 \\
\hline 5.0 & 5.4 \\
\hline 6.4 & 5.8 \\
\hline 8.0 & 6.1 \\
\hline 4.4 & 5.2 \\
\hline 100 & \\
\hline & 100 \\
\hline
\end{tabular}

$\begin{array}{lllll}1.5-1.0-0.5 & 0 & 0.5 & 1.0 & 1.5\end{array}$

SMD 3

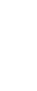

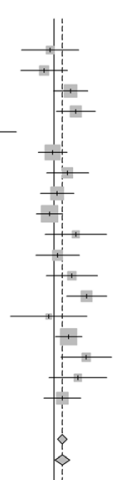

SMD $\quad 95 \% \mathrm{Cl} W$, fixed $W$, random

$0.10[-0.81,0.61]$ $\begin{array}{lll}-0.10[-0.81,0.61] & 2.7 & 4.2 \\ -0.25[-0.83,0.33] & 4.1 & 5.2 \\ 0.42[-0.010 .84] & 7.5 & 6.9\end{array}$ $0.54[0.06,1.03] \quad 5.9 \quad 6.9$ $\begin{array}{rrr}0.54[0.06,1.03] & 5.9 & 6.2 \\ -1.99[-3.03,-0.94] & 1.3 & 25\end{array}$ $\begin{array}{lrr}-0.03[-0.39,0.33] & 10.6 & 2.5 \\ 0.74\end{array}$ $0.34[-0.19,0.86] \quad 5.0 \quad 5.8$ $0.08[-0.34,0.50] \quad 7.9 \quad 7.0$

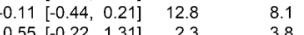
$0.55[-0.22,1.31] \quad 2.3 \quad 3.8$

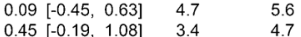
$0.82[0.32,1.32] \quad 5.5 \quad 6.1$ $0.13[-1.08,0.82] \quad 1.5 \quad 2.8$ $0.36[0.03,0.70] \quad 12.5 \quad 8.0$ $\begin{array}{lll}0.81[0.17,1.44] & 3.4 & 4.8 \\ 0.60[-0.13,1.32] & 2.6 & 4.1\end{array}$ $\begin{array}{lll}0.60[-0.13,1.32] & 2.6 & 4.1 \\ 0.21[-0.25,0.67] & 6.5 & 6.5\end{array}$

$0.21[0.09,0.32] \quad 100$ $0.21[0.02,0.39]$

100 
Table 3 Publication bias analysis in the sum of included studies, and after exclusion of paediatric/adolescent studies or studies of morbidly obese adults subjected to bariatric surgery or both

\begin{tabular}{lllll}
\hline Comparison & All studies & $\begin{array}{l}\text { Excluding paediatric/ } \\
\text { adolescent studies }\end{array}$ & $\begin{array}{l}\text { Excluding bariatric } \\
\text { studies }\end{array}$ & $\begin{array}{l}\text { Excluding both paediatric/adolescent } \\
\text { and bariatric studies }\end{array}$ \\
\hline NAFLD vs control & 0.98 & 0.98 & 0.97 & 0.99 \\
SS vs control & 0.13 & 0.13 & 0.12 & 0.12 \\
NASH vs control & 0.28 & 0.28 & 0.13 & 0.13 \\
NASH vs SS & 0.70 & 0.33 & 0.20 & 0.82 \\
\hline
\end{tabular}

Data are presented as $p$ values as derived from Egger's regression. If $p>0.05$, no publication bias is present

( $n=7$; ESM Fig. 3$)$ or both ( $n=10$; Fig. 3), there were only minimal changes in the comparisons between groups. Heterogeneity among studies remained generally moderate-to-severe ( $I^{2}$ ranged from $59.7 \%$ to $78.5 \%$ ), apart from the comparison between SS and NASH, which did not remain significant after the exclusion of paediatric studies $\left(I^{2}=21.9 \%\right)$, or both paediatric/adolescent and bariatric studies $\left(I^{2}=9.5 \%\right)$ (Fig. 3 and ESM Figs 2 and 3). No sign of publication bias was detected after excluding paediatric/adolescent or bariatric studies or both ( $p>0.05$ for all comparisons; Table 3). The results remained essentially unchanged when we used MD instead of SMD in the above-mentioned analyses (ESM Fig. 4).

To further investigate the impact of the preselected characteristics on leptin SMD, a random-effects meta-regression analysis was performed. BMI was inversely associated with leptin SMD, when comparing the following groups: (1) NASH patients vs controls $\left(\beta=-0.027, p=0.021, R^{2}=\right.$ $32.7 \%)$ and (2) NAFLD patients vs controls $(\beta=-0.031, p=$ $0.014, R^{2}=26.5 \%$ ). BMI was not associated with leptin SMD in the comparisons between SS and NASH patients (Table 4). Bubble plots demonstrating leptin SMD across different BMI levels in different comparisons between groups are depicted in ESM Fig. 5. When bariatric studies were excluded, BMI was not significantly associated with leptin SMD. The performance of liver biopsy on the controls was inversely associated with leptin SMD when comparing NASH patients with the controls ( $\left.\beta=-0.54, p=0.031, R^{2}=31.5 \%\right)$, whereas it had no effect in the other three comparisons (Table 4). The performance of liver biopsy on controls was not associated with leptin SMD when comparing NASH patients and controls, after excluding bariatric studies. Sex, age, HOMA-IR, type 2 diabetes and inclusion of NASH-related cirrhosis within the NASH group were not associated with leptin SMD (ESM Table 3).

\section{Discussion}

This meta-analysis demonstrated that high levels of leptin in the circulation were associated with the severity of NAFLD; controls had lower leptin levels than SS, NASH or NAFLD patients, and SS patients had lower leptin levels than NASH patients. The sensitivity analyses did not essentially change these findings.

The meta-regression analysis revealed that BMI was inversely associated with leptin SMD when comparing patients with NAFLD (or NASH) and controls, and that this association was no longer significant after the exclusion of morbidly obese patients. This may be partly attributed to differences in BMI between non-morbidly obese and morbidly obese adults subjected to bariatric surgery (Table 4 ). In any case, the magnitude of the association ( $\beta$ ), although statistically significant, was low $(-0.031$ and -0.027 , respectively), thereby possibly being of low clinical significance. The performance of liver biopsy in controls was also inversely associated with leptin SMD when comparing NASH patients and controls, and this association also lost its significance after the exclusion of morbidly obese patients. This seems reasonable given that, except for morbidly obese patients (and controls) subjected to bariatric surgery, liver biopsy in controls was performed only in two studies [29,34]; thus, when the majority of studies were excluded, the remaining two did not significantly add to the between-study heterogeneity. Leptin SMD was not associated with sex, age, HOMA-IR, type 2 diabetes or the inclusion of NASH-related cirrhosis within NASH group.

Experimental data have shown that leptin deficiency may lead to hepatic steatosis (e.g. in $o b / o b$ mice), which can be reversed by leptin replacement [9]. On the other hand, excess leptin may contribute to hepatic inflammation and fibrosis $[8$, 9]. However, this potential dual role of leptin cannot be proven by this meta-analysis of cross-sectional studies and it remains to be elucidated in large prospective cohort studies with paired biopsies.

Although liver biopsy is considered the gold standard for the assessment of liver fat, inflammation and fibrosis, there is a need for less invasive diagnostic techniques to facilitate diagnosis and avoid complications related to liver biopsy [10]. This meta-analysis cannot answer the question of whether circulating leptin could serve as a surrogate non-invasive marker for NASH, because this answer requires studies of different design. However, this meta-analysis could be a 
Fig. 3 Forest plots presenting the quantitative synthesis of circulating leptin level, comparing the following groups after excluding studies on paediatric/adolescent populations and morbidly obese adults subjected to bariatric surgery:

NAFLD patients vs controls (a); SS patients vs controls (b);

NASH patients vs controls (c);

NASH patients vs SS patients (d). $\mathrm{W}$, weight a

Authors (year) [ref.] Total Mean ${ }^{\text {NAFLD }}$ SD Total Mean ${ }^{\text {Control }}$

$\begin{array}{lllllll}\text { Angulo et al (2004) [17] } & 68 & 19.7 & 11.7 & 68 & 19.1 & 13.0\end{array}$ $\begin{array}{lllllll}\text { Chalasani et al (2003) [21] } & 26 & 21.0 & 13.0 & 20 & 18.0 & 12.0\end{array}$ $\begin{array}{lllllrr}\text { Chalasani et al (2003) [21] } & 26 & 21.0 & 13.0 & 20 & 18.0 & 12.0 \\ \text { Chitturi et al (2002) [23] } & 47 & 22.9 & 13.1 & 47 & 10.5 & 5.8\end{array}$ $\begin{array}{llllllr}\text { Chitturi et al (2002) [23] } & 47 & 22.9 & 13.1 & 47 & 10.5 & 5.8 \\ \text { Dasarathy et al (2011) [24] } & 10 & 30.1 & 22.9 & 16 & 15.9 & 14.9\end{array}$ $\begin{array}{lrrrrrr}\text { Dasarathy et al (2011) [24] } & 10 & 30.1 & 22.9 & 16 & 15.9 & 14.9 \\ \text { Giannini et al (1999) [26] } & 6 & 5.7 & 4.9 & 6 & 4.8 & 1.7\end{array}$ $\begin{array}{lrrrrrr}\text { Giannini et al (1999) [26] } & 6 & 5.7 & 4.9 & 6 & 4.8 & 1.7 \\ \text { Gonciarz et al (2012) [27] } & 16 & 11.7 & 6.4 & 15 & 8.1 & 15.3\end{array}$ $\begin{array}{lrrrrrr}\text { Gonciarz et al (2012) [27] } & 16 & 11.7 & 6.4 & 15 & 8.1 & 15.3\end{array}$ $\begin{array}{lllllll}\text { Haukeland et al (2012) [28] } & 119 & 18.7 & 14.6 & 93 & 6.9 & 5.9\end{array}$ $\begin{array}{lllllll}\text { Huang et al (2008) [29] } & 30 & 10.9 & 3.4 & 30 & 5.6 & 2.0\end{array}$ $\begin{array}{lrlllll}\text { Hui et al (2004) [30] } & 109 & 17.5 & 13.2 & 53 & 11.5 & 6.6\end{array}$ $\begin{array}{lllllll}\text { Lemoine et al (2009) [34] } & 74 & 14.1 & 11.1 & 10 & 5.8 & 6.6\end{array}$ $\begin{array}{lllllll}\text { Munoz et al (2009) [37] } & 52 & 15.6 & 12.8 & 49 & 4.3 & 2.6\end{array}$ $\begin{array}{lllllll}\text { Musso et al (2005) [38] } & 25 & 2.1 & 4.0 & 25 & 2.5 & 3.4\end{array}$ Pagano et al (2005) [40] $\quad \begin{array}{llllll}17 & 9.1 & 4.7 & 20 & 9.9 & 5.3\end{array}$

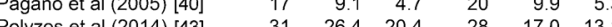

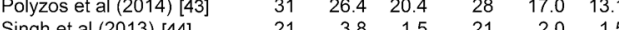
$\begin{array}{lllllll}\text { Singh et al (2013) [44] } & 21 & 3.8 & 1.5 & 21 & 2.0 & 1.5\end{array}$ $\begin{array}{lrrrrrr}\text { Swellam et al (2012) [45] } & 90 & 14.3 & 6.6 & 30 & 5.9 & 3.0 \\ \text { Tungtrongchitr et al (2006) [46] } 20 & 17.1 & 9.2 & 60 & 5.1 & 13.7\end{array}$ $\begin{array}{lrrrrrr}\text { Tungtrongchitr et al (2006) [46] } & 20 & 17.1 & 9.2 & 60 & 5.1 & 13.7 \\ \text { Uygun et al (2000) [47] } & 49 & 6.6 & 4.9 & 30 & 4.0 & 2.2\end{array}$ $\begin{array}{llrrrrr}\text { Uygun et al (2000) [4] } & 49 & 6.6 & 4.9 & 30 & 4.0 & 2.2\end{array}$ $\begin{array}{lllllll}\text { Wong et al (2006) [48] } & 80 & 25.0 & 19.0 & 41 & 17.0 & 20.0\end{array}$ $\begin{array}{llllllr}\text { Yalniz et al (2006) [49] } & 37 & 15.5 & 4.8 & 25 & 10.3 & 2.5\end{array}$

Fixed effect model $\quad 927$

Random effects model

687

Heterogeneity: $I^{2}=77.0 \%, \tau^{2}=0.22, p<0.0001$

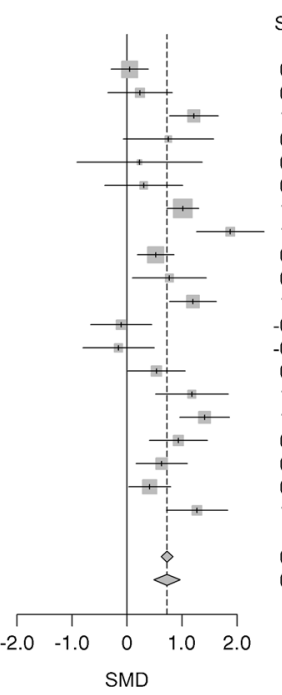

SMD $\quad 95 \% \mathrm{Cl} \quad \mathrm{W}$, fixed $\quad \mathrm{W}$, random

$\begin{array}{lll}(\%) & (\%)\end{array}$

$\begin{array}{ll}10.1 & 6.0\end{array}$

$\begin{array}{lll}0.23[-0.35,0.82] & 3.3 & 4.8 \\ 1.21[0.77,1.66] & 5.9 & 5.5\end{array}$

$0.75[-0.07,1.57] \quad 1.7 \quad 3.8$

$0.23[-0.91,1.36] \quad 0.9 \quad 2.7$

$0.30[-0.41,1.01] \quad 2.3 \quad 4.3$

$\begin{array}{rrr}1.01[0.72,1.30] & 13.8 & 6.2 \\ 1.88[1.26,2.49] & 3.0 & 4.7\end{array}$

$0.52[0.19,0.85] \quad 10.3 \quad 6.0$

$0.77[0.10,1.44] \quad 2.5 \quad 4.4$

$1.20[0.77,1.62] \quad 6.3 \quad 5.6$

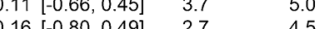

$0.54[0.01,1.06]-4.2 \quad 4.5$

$1.18[0.52,1.84]-2.6 \quad 5.1$

$1.41[0.96,1.86] \quad 5.6 \quad 5.5$

$0.93[0.40,1.46] \quad 4.1 \quad 5.1$

$0.63[0.16,1.09] \quad 5.3 \quad 5.4$

$0.41[0.03,0.79] \quad 7.9 \quad 5.8$

5.0

$0.73[0.62,0.83] \quad 100$

100

b

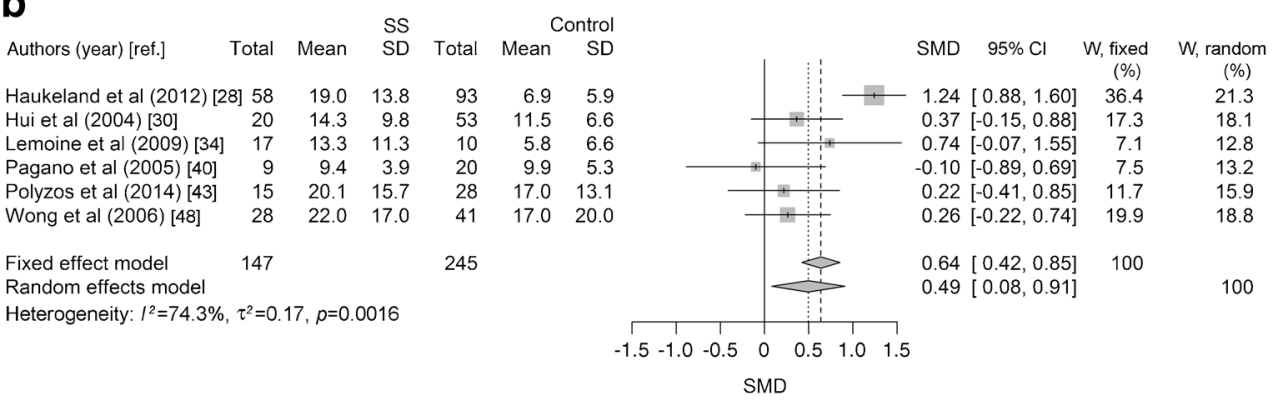

C

\begin{tabular}{lrrrrrr} 
& \multicolumn{3}{c}{ NASH } & \multicolumn{2}{r}{ Control } \\
Authors (year) [ref.] & Total & Mean & SD & Total & Mean & SD \\
& & & & & & \\
Chalasani et al (2003) [21] & 26 & 21.0 & 13.0 & 20 & 18.0 & 12.0 \\
Chitturi et al (2002) [23] & 47 & 22.9 & 13.1 & 47 & 10.5 & 5.8 \\
Dasarathy et al (2011) [24] & 10 & 30.1 & 22.9 & 16 & 15.9 & 14.9 \\
Giannini et al (1999) [26] & 6 & 5.7 & 4.9 & 6 & 4.8 & 1.7 \\
Gonciarz et al (2012) [27] & 16 & 11.7 & 6.4 & 15 & 8.1 & 15.3 \\
Haukeland et al (2012) [28] & 61 & 18.5 & 15.3 & 93 & 6.9 & 5.9 \\
Hui et al (2004) [30] & 48 & 18.9 & 14.6 & 53 & 11.5 & 6.6 \\
Lemoine et al (2009) [34] & 57 & 14.3 & 11.1 & 10 & 5.8 & 6.6 \\
Munoz et al (2009) [37] & 52 & 15.6 & 12.8 & 49 & 4.3 & 2.6 \\
Musso et al (2005) [38] & 25 & 2.1 & 4.0 & 25 & 2.5 & 3.4 \\
Pagano et al (2005) [40] & 8 & 8.8 & 4.8 & 20 & 9.9 & 5.3 \\
Polyzos et al (2014) [43] & 16 & 32.2 & 22.9 & 28 & 17.0 & 13.1 \\
Singh et al (2013) [44] & 21 & 3.8 & 1.5 & 21 & 2.0 & 1.5 \\
Tungtrongchitr et al (2006) [46] 20 & 17.1 & 9.2 & 60 & 5.1 & 13.7 \\
Uygun et al (2000) [47] & 49 & 6.6 & 4.9 & 30 & 4.0 & 2.2 \\
Wong et al (2006) [48] & 52 & 26.0 & 20.0 & 41 & 17.0 & 20.0 \\
Yalniz et al (2006) [49] & 37 & 15.5 & 4.8 & 25 & 10.3 & 2.5 \\
& & & & & &
\end{tabular}

Fixed effect model $\quad 551 \quad 559$

Random effects model

Heterogeneity: $I^{2}=59.7 \%, \tau^{2}=0.11, p=0.0009$

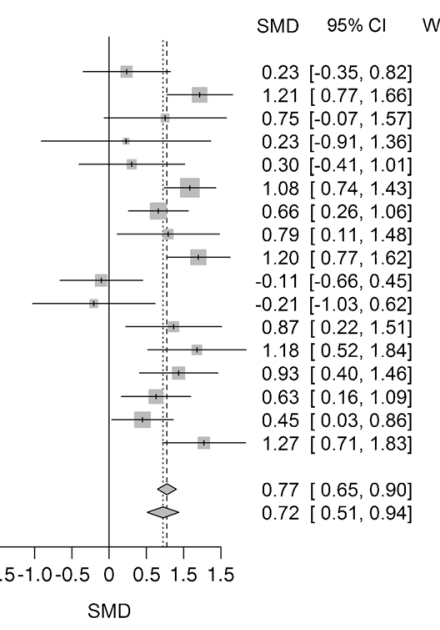

$\begin{array}{cc}\begin{array}{c}\text { W, fixed } \\ (\%)\end{array} & \begin{array}{c}\text { W, random } \\ (\%)\end{array} \\ 4.8 & 5.8 \\ 8.4 & 7.2 \\ 2.4 & 4.1 \\ 1.3 & 2.6 \\ 3.2 & 4.8 \\ 13.7 & 8.1 \\ 10.1 & 7.6 \\ 3.5 & 5.0 \\ 9.0 & 7.3 \\ 5.3 & 6.1 \\ 2.4 & 4.1 \\ 4.0 & 5.3 \\ 3.7 & 5.2 \\ 5.9 & 6.3 \\ 7.5 & 6.9 \\ 9.5 & 7.4 \\ 5.3 & 6.1 \\ & \\ 100 & \\ & 100\end{array}$

d Authors (year) [ref.] Total Mean $\begin{array}{llll}\text { NASH } & \text { SS } \\ \text { Total Mean } & \text { SD }\end{array}$

$\begin{array}{lllllll}\text { Canbakan et al (2008) [19] } & 35 & 52.6 & 36.8 & 17 & 61.8 & 36.3\end{array}$ $\begin{array}{lllllll}\text { Charlton et al (2008) [22] } & 25 & 33.9 & 20.6 & 53 & 24.5 & 15.2\end{array}$ $\begin{array}{lllllll}\text { Haukeland et al (2012) [28] } & 61 & 18.5 & 15.3 & 58 & 19.0 & 13.8\end{array}$ $\begin{array}{llllllr}\text { Hui et al (2004) [30] } & 48 & 18.9 & 14.6 & 20 & 14.3 & 9.8\end{array}$ $\begin{array}{lllllll}\text { Lemoine et al (2009) [34] } & 57 & 14.3 & 11.1 & 17 & 13.3 & 11.3\end{array}$ $\begin{array}{lrrrrrr}\text { Lemoine et al (2009) [34] } & 57 & 14.3 & 11.1 & 17 & 13.3 & 11.3 \\ \text { Pagano et al (2005) [40] } & 8 & 8.8 & 4.8 & 9 & 9.4 & 3.9\end{array}$ $\begin{array}{lrrrrrr}\text { Pagano et al (2005) [40] } & 8 & 8.8 & 4.8 & 9 & 9.4 & 3.9 \\ \text { Petta et al (2012) [41] } & 71 & 18.2 & 13.8 & 71 & 12.8 & 15.6\end{array}$ $\begin{array}{lllllll}\text { Petta et al }(2012)[41] & 71 & 18.2 & 13.8 & 71 & 12.8 & 15.6\end{array}$ $\begin{array}{lllllll}\text { Polyzos et al (2014) [43] } & 16 & 32.2 & 22.9 & 15 & 20.1 & 15.7 \\ \text { Wong et al (2006) [48] } & 52 & 26.0 & 20.0 & 28 & 22.0 & 17.0\end{array}$

$\begin{array}{lll}\text { Fixed effect model } \quad 373 & 288\end{array}$

Random effects model

Heterogeneity: $I^{2}=9.5 \%, \tau^{2}=0.01, p=0.356$

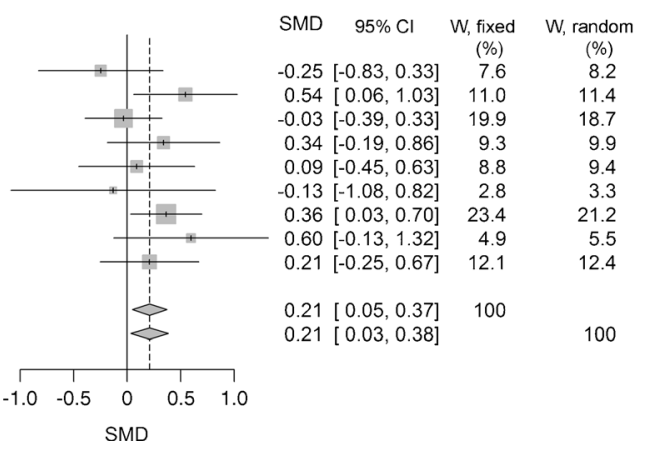


Table 4 Random-effects meta-regression analysis for BMI and the performance of liver biopsy in the controls in the sum of included studies, and after exclusion of paediatric/adolescent studies or studies of morbidly obese adults subjected to bariatric surgery or both

\begin{tabular}{|c|c|c|c|c|}
\hline Analysis & All studies & $\begin{array}{l}\text { Excluding paediatric/ } \\
\text { adolescent studies }\end{array}$ & $\begin{array}{l}\text { Excluding bariatric } \\
\text { studies }\end{array}$ & $\begin{array}{l}\text { Excluding both paediatric/adolescent } \\
\text { and bariatric studies }\end{array}$ \\
\hline \multicolumn{5}{|l|}{ BMI } \\
\hline \multicolumn{5}{|c|}{ NAFLD vs control } \\
\hline$R^{2}(\%)$ & 26.5 & 26.4 & 8.3 & 8.5 \\
\hline$\beta$ & -0.031 & -0.031 & -0.063 & -0.065 \\
\hline$p$ value & 0.014 & 0.015 & 0.17 & 0.18 \\
\hline \multicolumn{5}{|c|}{ SS vs control } \\
\hline$R^{2}(\%)$ & 21.6 & 21.6 & 0.0 & 0.0 \\
\hline$\beta$ & -0.020 & -0.020 & -0.054 & -0.054 \\
\hline$p$ value & 0.161 & 0.161 & 0.677 & 0.677 \\
\hline \multicolumn{5}{|c|}{ NASH vs control } \\
\hline$R^{2}(\%)$ & 32.7 & 32.7 & 0.0 & 0.0 \\
\hline$\beta$ & -0.027 & -0.027 & 0.012 & 0.012 \\
\hline$p$ value & 0.021 & 0.021 & 0.808 & 0.808 \\
\hline \multicolumn{5}{|c|}{ NASH vs SS } \\
\hline$R^{2}(\%)$ & 0.0 & 0.0 & 0.0 & 99.9 \\
\hline$\beta$ & -0.004 & 0.001 & 0.024 & 0.086 \\
\hline$p$ value & 0.658 & 0.992 & 0.644 & 0.050 \\
\hline \multicolumn{5}{|c|}{ Liver biopsy in the controls } \\
\hline \multicolumn{5}{|c|}{ NAFLD vs control } \\
\hline$R^{2}(\%)$ & 0.0 & 0.0 & 10.2 & 9.7 \\
\hline$\beta$ & -0.136 & -0.137 & 0.653 & 0.650 \\
\hline$p$ value & 0.633 & 0.639 & 0.103 & 0.113 \\
\hline \multicolumn{5}{|c|}{ SS vs control } \\
\hline$R^{2}(\%)$ & 3.5 & 3.5 & 0.0 & 0.0 \\
\hline$\beta$ & -0.264 & -0.264 & 0.286 & 0.286 \\
\hline$p$ value & 0.412 & 0.412 & 0.667 & 0.667 \\
\hline \multicolumn{5}{|c|}{ NASH vs control } \\
\hline$R^{2}(\%)$ & 31.5 & 31.5 & 0.0 & 0.0 \\
\hline$\beta$ & -0.538 & -0.538 & 0.044 & 0.044 \\
\hline$p$ value & 0.031 & 0.031 & 0.931 & 0.931 \\
\hline \multicolumn{5}{|c|}{ NASH vs SS } \\
\hline$R^{2}(\%)$ & 0.0 & 0.0 & 5.6 & 0.0 \\
\hline$\beta$ & -0.180 & -0.023 & -0.796 & -0.012 \\
\hline$p$ value & 0.508 & 0.895 & 0.098 & 0.968 \\
\hline
\end{tabular}

Data are presented as $R^{2}(\%), \beta$ and $p$ value for $\beta$

trigger for the design of clinical trials investigating whether leptin, alone or in combination with other variables, could serve as non-invasive index. There are limited data on whether leptin could be used as a non-invasive biomarker for NAFLD, and more research in this area is needed.

The results of this meta-analysis do not warrant the design of trials with recombinant leptin in NASH patients with normoleptinaemia or hyperleptinaemia. NASH patients have high leptin levels and, based on experimental studies, leptin administration might have an adverse effect on liver fibrosis, thereby possibly worsening the prognosis of the disease. In line, to the best of our knowledge, there is no ongoing study evaluating the effect of recombinant leptin administration in NASH patients with normoleptinaemia or hyperleptinaemia. On the contrary, NAFLD patients with hypoleptinaemia, being the minority of NAFLD patients, might possibly benefit from leptin replacement. The first evidence comes from uncontrolled studies with lipodystrophic patients with NAFLD [9]; leptin seems to be a highly effective treatment for NASH in hypoleptinaemic lipodystrophic patients, but this remains to be fully proven by future long-term and well-controlled studies. 
This meta-analysis has certain limitations. First, all included studies were observational (cross-sectional), therefore a cause-effect relationship between circulating leptin and NAFLD severity cannot be shown. There are limited data from longitudinal cohort studies providing evidence on leptin changes when NAFLD progresses or regresses over time [9]. These studies have been inconclusive and are primarily limited by the high dropout rates, because of the need for repeat liver biopsy. Second, the between-study heterogeneity limits the reliability of the results, which should be cautiously interpreted, especially in regard to BMI differences between groups being compared. Third, we used BMI and not waist circumference as a measure of adiposity, mainly because waist circumference was not recorded in many of the included studies. However, since leptin reflects total body fat, BMI can serve as a sufficient measure of adiposity. Fourth, by excluding unpublished studies or abstracts from conferences, a publication bias might exist. Excluding such studies, however, is essential to avoid introduction of low-quality data, since the quality of unpublished data and abstracts cannot be fully reviewed [50]. Fifth, there is no well-validated quality-assessment tool for cross-sectional studies. We used the most commonly utilised scale for observational studies (NOS), aiming to exclude studies with very poor quality. Sixth, some studies included patients with NASH-related cirrhosis in the NASH group; we did not exclude these studies, because we considered that the selection bias might outweigh the effect that a minority of patients with NASH-related cirrhosis on circulating leptin levels might have, if any. Furthermore, most studies included only a small sample of cirrhotic patients (Table 1). Patients with NASH-related cirrhosis were not examined separately in this meta-analysis, since none of the studies examined these patients as a separate group. Leptin levels have been elevated in the majority of studies examining patients with cirrhosis of other or mixed (including NASH-related) aetiology [9]. Seventh, the lack of liver biopsy in controls was not an exclusion criterion because a selection bias might have been committed; since liver biopsy in controls meets ethical considerations, controls were recruited on the basis of normal liver ultrasound and serum aminotransferases levels in most studies. On the other hand, another type of selection bias may also be committed in most studies in which controls were subjected to liver biopsy, because they were selected populations (e.g. morbidly obese patients subjected to bariatric surgery or patients subjected to laparoscopic cholecystectomy or hepatectomy for benign tumours) (Table 1). Eighth, the quality of the included case-control studies might be also limited due to the fact that only three studies explicitly mentioned that the controls were drawn from the same pool as the cases (Table 1). Ninth, we did not examine leptin levels separately for steatosis grade or inflammation or fibrosis stage, since only a minority of studies provided data for specific histological lesions; this was further limited by the studies' different histological definitions and group division. Finally, none of the included studies reported a risk ratio for different levels of leptin, so we were not able to evaluate a potential non-linear association of leptin.

In conclusion, this meta-analysis demonstrated that a high level of circulating leptin was associated with the severity of NAFLD. These results are consistent with the notion of an anti-steatotic action of leptin during the initial stages of NAFLD and a pro-inflammatory and fibrotic action during disease progression. This speculation, which has been shown in experimental models of NAFLD, remains to be elucidated in humans by prospective cohort studies with paired biopsies. This meta-analysis also warrants further studies examining the role of leptin, alone or in combination with other variables, as a non-invasive biomarker for NAFLD.

Acknowledgements We are sincerely grateful to P. Angulo (Mayo Clinic College of Medicine, USA), M. Argentou (University of Patras, Greece), N. Chalasani (Indiana University School of Medicine, USA), S. Chitturi (University of Sydney, New Zealand), S. Dasarathy (Cleveland Clinic, USA), E. Giannini (University of Genova, Italy), M. Gonciarz (St Barbara's Main District Hospital, Poland), J. W. Haukeland (University of Oslo, Norway), S. R. Kashyap (Cleveland Clinic, USA), M. V. Machado (Hospital of Santa Maria, Portugal), R. W. O'Rourke and D. Marks (Oregon Health and Science University, USA), S. Petta (University of Palermo, Italy), I. Pirvulescu (Fundeni Clinical Institute, Romania), L. Serfaty and M. Lemoine (University of Pierre and Marie Curie, France), V. W. Wong and H. L. Chan (Chinese University of Hong Kong, China) and M. Yalniz (Firat University, Turkey) for their willingness to provide additional data on their studies, regarded as being invaluable to this work.

Duality of interest CSM has served as a consultant for Astra Zeneca and has received research support through his Institution from Amgen and a lecture fee from Aegerion. All other authors declare that there is no duality of interest associated with their contribution to this manuscript.

Contribution statement SAP, JK and CSM were responsible for the study concept and design. SAP, DDR and MFV acquired data. Data were interpreted by SAP and KNA and statistical analysis was carried out by KNA. The manuscript was drafted by SAP and critically revised for important intellectual content by all authors. All authors approved the final version of the manuscript to be published. SAP is the guarantor of this work.

\section{References}

1. Polyzos SA, Kountouras J, Zavos C (2009) Nonalcoholic fatty liver disease: the pathogenetic roles of insulin resistance and adipocytokines. Curr Mol Med 72:299-314

2. Vernon G, Baranova A, Younossi ZM (2011) Systematic review: the epidemiology and natural history of non-alcoholic fatty liver disease and non-alcoholic steatohepatitis in adults. Aliment Pharmacol Ther 34:274-285

3. Kelesidis T, Kelesidis I, Chou S, Mantzoros CS (2010) Narrative review: the role of leptin in human physiology: emerging clinical applications. Ann Intern Med 152:93-100

4. Moon HS, Dalamaga M, Kim SY et al (2013) Leptin's role in lipodystrophic and nonlipodystrophic insulin-resistant and diabetic individuals. Endocr Rev 34:377-412 
5. Mantzoros CS, Magkos F, Brinkoetter M et al (2011) Leptin in human physiology and pathophysiology. Am J Physiol Endocrinol Metab 301:E567-E584

6. Dalamaga M, Chou SH, Shields K, Papageorgiou P, Polyzos SA, Mantzoros CS (2013) Leptin at the intersection of neuroendocrinology and metabolism: current evidence and therapeutic perspectives. Cell Metab 18:29-42

7. Polyzos SA, Kountouras J, Zavos C, Deretzi G (2011) The potential adverse role of leptin resistance in nonalcoholic fatty liver disease: a hypothesis based on critical review of literature. J Clin Gastroenterol 45:50-54

8. Imajo K, Fujita K, Yoneda M et al (2012) Hyperresponsivity to lowdose endotoxin during progression to nonalcoholic steatohepatitis is regulated by leptin-mediated signaling. Cell Metab 16:44-54

9. Polyzos SA, Kountouras J, Mantzoros CS (2015) Leptin in nonalcoholic fatty liver disease: a narrative review. Metabolism 64:60-78

10. Polyzos SA, Mantzoros CS (2014) Necessity for timely noninvasive diagnosis of nonalcoholic fatty liver disease. Metabolism 63:161-167

11. Polyzos SA, Mantzoros CS (2015) Leptin in health and disease: facts and expectations at its twentieth anniversary. Metabolism 64:5-12

12. Stroup DF, Berlin JA, Morton SC et al (2000) Meta-analysis of observational studies in epidemiology: a proposal for reporting. Meta-analysis Of Observational Studies in Epidemiology (MOOSE) group. JAMA 283:2008-2012

13. Polyzos SA, Toulis KA, Goulis DG, Zavos C, Kountouras J (2011) Serum total adiponectin in nonalcoholic fatty liver disease: a systematic review and meta-analysis. Metabolism 60:313-326

14. Brunt EM, Janney CG, Di Bisceglie AM, Neuschwander-Tetri BA, Bacon BR (1999) Nonalcoholic steatohepatitis: a proposal for grading and staging the histological lesions. Am J Gastroenterol 94:2467-2474

15. Kleiner DE, Brunt EM, Van NM et al (2005) Design and validation of a histological scoring system for nonalcoholic fatty liver disease. Hepatology 41:1313-1321

16. Moher D, Liberati A, Tetzlaff J, Altman DG (2009) Preferred reporting items for systematic reviews and meta-analyses: the PRISMA statement. Ann Intern Med 151:264-269

17. Angulo P, Alba LM, Petrovic LM, Adams LA, Lindor KD, Jensen MD (2004) Leptin, insulin resistance, and liver fibrosis in human nonalcoholic fatty liver disease. J Hepatol 41:943-949

18. Argentou M, Tiniakos DG, Karanikolas M et al (2009) Adipokine serum levels are related to liver histology in severely obese patients undergoing bariatric surgery. Obes Surg 19:1313-1323

19. Canbakan B, Tahan V, Balci H et al (2008) Leptin in nonalcoholic fatty liver disease. Ann Hepatol 7:249-254

20. Cayon A, Crespo J, Mayorga M, Guerra A, Pons-Romero F (2006) Increased expression of $\mathrm{Ob}-\mathrm{Rb}$ and its relationship with the overexpression of TGF- $\beta 1$ and the stage of fibrosis in patients with nonalcoholic steatohepatitis. Liver Int 26:1065-1071

21. Chalasani N, Crabb DW, Cummings OW et al (2003) Does leptin play a role in the pathogenesis of human nonalcoholic steatohepatitis? Am J Gastroenterol 98:2771-2776

22. Charlton M, Angulo P, Chalasani N et al (2008) Low circulating levels of dehydroepiandrosterone in histologically advanced nonalcoholic fatty liver disease. Hepatology 47:484-492

23. Chitturi S, Farrell G, Frost L et al (2002) Serum leptin in NASH correlates with hepatic steatosis but not fibrosis: a manifestation of lipotoxicity? Hepatology 36:403-409

24. Dasarathy S, Yang Y, McCullough AJ, Marczewski S, Bennett C, Kalhan SC (2011) Elevated hepatic fatty acid oxidation, high plasma fibroblast growth factor 21, and fasting bile acids in nonalcoholic steatohepatitis. Eur J Gastroenterol Hepatol 23:382-388
25. Fitzpatrick E, Dew TK, Quaglia A, Sherwood RA, Mitry RR, Dhawan A (2012) Analysis of adipokine concentrations in paediatric non-alcoholic fatty liver disease. Pediatr Obes 7:471-479

26. Giannini E, Botta F, Cataldi A et al (1999) Leptin levels in nonalcoholic steatohepatitis and chronic hepatitis C. Hepatogastroenterology 46:2422-2425

27. Gonciarz M, Bielanski W, Partyka R et al (2013) Plasma insulin, leptin, adiponectin, resistin, ghrelin, and melatonin in nonalcoholic steatohepatitis patients treated with melatonin. J Pineal Res 54:154-161

28. Haukeland JW, Dahl TB, Yndestad A et al (2012) Fetuin A in nonalcoholic fatty liver disease: in vivo and in vitro studies. Eur J Endocrinol 166:503-510

29. Huang XD, Fan Y, Zhang H et al (2008) Serum leptin and soluble leptin receptor in non-alcoholic fatty liver disease. World $\mathrm{J}$ Gastroenterol 14:2888-2893

30. Hui JM, Hodge A, Farrell GC, Kench JG, Kriketos A, George J (2004) Beyond insulin resistance in NASH: TNF-alpha or adiponectin? Hepatology 40:46-54

31. Kashyap SR, Diab DL, Baker AR et al (2009) Triglyceride levels and not adipokine concentrations are closely related to severity of nonalcoholic fatty liver disease in an obesity surgery cohort. Obesity (Silver Spring) 17:1696-1701

32. Koehler E, Swain J, Sanderson S, Krishnan A, Watt K, Charlton M (2012) Growth hormone, dehydroepiandrosterone and adiponectin levels in non-alcoholic steatohepatitis: an endocrine signature for advanced fibrosis in obese patients. Liver Int 32:279-286

33. Le D, Marks D, Lyle E et al (2007) Serum leptin levels, hepatic leptin receptor transcription, and clinical predictors of non-alcoholic steatohepatitis in obese bariatric surgery patients. Surg Endosc 21:1593-1599

34. Lemoine M, Ratziu V, Kim M et al (2009) Serum adipokine levels predictive of liver injury in non-alcoholic fatty liver disease. Liver Int 29:1431-1438

35. Machado MV, Coutinho J, Carepa F, Costa A, Proenca H, CortezPinto H (2012) How adiponectin, leptin, and ghrelin orchestrate together and correlate with the severity of nonalcoholic fatty liver disease. Eur J Gastroenterol Hepatol 24:1166-1172

36. Manco M, Giordano U, Turchetta A et al (2009) Insulin resistance and exercise capacity in male children and adolescents with nonalcoholic fatty liver disease. Acta Diabetol 46:97-104

37. Munoz LE, Cordero P, Torres L, Sauceda AY, Flores JP, Segura JJ (2009) Adipokines in a group of Mexican patients with nonalcoholic steatohepatitis. Ann Hepatol 8:123-128

38. Musso G, Gambino R, Durazzo M et al (2005) Adipokines in NASH: postprandial lipid metabolism as a link between adiponectin and liver disease. Hepatology 42:1175-1183

39. Nobili V, Manco M, Ciampalini P et al (2006) Leptin, free leptin index, insulin resistance and liver fibrosis in children with nonalcoholic fatty liver disease. Eur J Endocrinol 155:735-743

40. Pagano C, Soardo G, Esposito W et al (2005) Plasma adiponectin is decreased in nonalcoholic fatty liver disease. Eur J Endocrinol 152:113-118

41. Petta S, Amato MC, Di Marco V et al (2012) Visceral adiposity index is associated with significant fibrosis in patients with nonalcoholic fatty liver disease. Aliment Pharmacol Ther 35:238-247

42. Pirvulescu I, Gheorghe L, Csiki I et al (2012) Noninvasive clinical model for the diagnosis of nonalcoholic steatohepatitis in overweight and morbidly obese patients undergoing bariatric surgery. Chirurgia (Bucur) 107:772-779

43. Polyzos SA, Kountouras J, Anastasilakis AD, Geladari EV, Mantzoros CS (2014) Irisin in patients with nonalcoholic fatty liver disease. Metabolism 63:207-217

44. Singh DK, Sakhuja P, Rastogi A, Singh A, Gondal R, Sarin SK (2013) Serum leptin levels correlate with body mass index but not with histologic disease severity in Indian patients with non-alcoholic steatohepatitis: a pilot study. Indian J Med Res 137:986-987 
45. Swellam M, Hamdy N (2012) Association of nonalcoholic fatty liver disease with a single nucleotide polymorphism on the gene encoding leptin receptor. IUBMB Life 64:180-186

46. Tungtrongchitr R, Treeprasertsuk S, Ei NN, Thepouyporn A, Phonrat B, Huntrup A (2006) Serum leptin concentrations in chronic hepatitis. J Med Assoc Thail 89:490-499

47. Uygun A, Kadayifci A, Yesilova Z et al (2000) Serum leptin levels in patients with nonalcoholic steatohepatitis. Am J Gastroenterol 95:3584-3589
48. Wong VW, Hui AY, Tsang SW et al (2006) Metabolic and adipokine profile of Chinese patients with nonalcoholic fatty liver disease. Clin Gastroenterol Hepatol 4:1154-1161

49. Yalniz M, Bahcecioglu IH, Ataseven H et al (2006) Serum adipokine and ghrelin levels in nonalcoholic steatohepatitis. Mediat Inflamm 2006:34295

50. Egger M, Juni P, Bartlett C, Holenstein F, Sterne J (2003) How important are comprehensive literature searches and the assessment of trial quality in systematic reviews? Empirical study. Health Technol Assess 7:1-76 